\title{
Infantile Perianal Pyramidal Protrusion: A Report of 8 New Cases and a Review of the Literature
}

\author{
N. Zavras ${ }^{a} \quad$ E. Christianakis ${ }^{b} \quad$ S. Tsamoudaki ${ }^{\text {b }}$ \\ K. Velaoras ${ }^{\mathrm{b}}$ \\ a'Third Department of Surgery, University Hospital 'ATTIKO', and \\ ${ }^{b}$ Pediatric Surgery Department, Penteli General Children's Hospital, \\ Athens, Greece
}

\section{Key Words}

Infantile perianal pyramidal protrusion - Constipation · Gender predilection

\begin{abstract}
Infantile perianal pyramidal protrusion (IPPP) is characterized by a protrusion located most often anterior to the anus. Three types of IPPPs are recognized: (a) constitutional, (b) acquired (mainly due to constipation), and (c) IPPPs due to lichen sclerosus et atrophicus. The aim of this study was to make a brief review on IPPPs from articles retrieved from PubMed, and to present our experience in this field. We conclude that awareness of pediatric surgeons and pediatricians of the condition is essential for the correct diagnosis of IPPP, and could help to avoid erroneous investigation of other lesions in this area.
\end{abstract}

\section{Introduction}

In 1996, Kayashima et al. [1] introduced the term infantile perianal pyramidal protrusion (IPPP) to describe a rare benign condition characterized by a pyramidal protrusion, and localized in the perineal median raphe anterior to the anus. These lesions are usually solitary and mostly observed in young females $[2,3]$. In previous reports, IPPPs have been described as skin tags/folds [4]. Herein, we present a case of IPPP and make a brief review of the current literature. 


\section{Case Presentations}

During a 5-year period (2007-2011), a diagnosis of IPPP was made in 8 ( 6 females and 2 males, aged 6 months to 13 years) healthy children. There was no suspicion of sexual abuse. In 7 patients, the IPPP was located anterior to the anus ( $\underline{\text { fig. } 1}$ fig. 2 ). Past history of 4 patients revealed the presence of IPPP since birth, and 3 of these patients had a family history of IPPP. In 2 other patients, the protrusion appeared 6 months and 3 years before consultation, respectively, while further 2 patients had a history of intermittent constipation. In these patients, the protrusion was resolved after appropriate treatment. IPPPs did not regress in the remaining 6 patients during a follow-up period of 6 months to 5 years. None of the patients underwent biopsy.

\section{Discussion}

The prevalence of IPPP is unknown. Till now, 99 cases of IPPP have been recorded in the literature, including those described as skin tags/folds [4] (table 1). Of interest is that most patients $(98 / 99)$ were females. The cause for gender predilection is obscure. A possible explanation is that the lesion is more noticeable in females [1]. Concerning the age of patients, IPPP is most often observed at birth [3]. This study supports the above findings, as $6 / 8$ patients were girls, and IPPP was observed at birth in $4 / 8$ patients.

Pathogenesis of IPPP is unclear. Kayashima et al. [1] suggested that IPPP is due to a mechanical stimulation of the perineum from wiping after defecation. However, this opinion does not apply to the presence of the lesion in neonates and infants whose stools are softer than those of older children. Currently, 3 types of IPPPs are recognized: (a) constitutional IPPPs that can sometimes be familial and/or congenital, (b) acquired IPPPs, and (c) IPPPs due to lichen sclerosus et atrophicus (LSA) [5, 6]. According to Mérigou et al. [7], constitutional IPPPs develop due to a weakness in the area of the perineum in females, which may explain why such conditions are seen mostly in girls. However, this hypothesis does not explain the presence of IPPPs in boys [8]. The congenital origin of IPPP is based on the discovery of these lesions in newborns and infants [3]. Furthermore, in certain cases (as in patients 1-3 of this study), the development of IPPP had to do with other members of the family [3, 6]. Konta et al. [3] suggested that congenital IPPP may be 'a remnant of a projected tip of the urogenital septum'. In the acquired type, constipation seems to play a role as IPPP regresses after appropriate management [7-9]. However, it is not clear whether regression is due to the treatment of constipation or constipation is a consequence of the development of IPPP [8]. The presence of diarrhea, fistulas, and fissures could also implicate the formation of IPPP because of mechanical stimulation of the perineum [5]. Cruces et al. [9] suggested that IPPP might be a peculiar form of LSA. Patrizi et al. [5] stated that in certain cases, IPPPs might be an early manifestation of LSA, although in other cases they might coexist with LSA. Typically, IPPP is presented as an expanded pyramidal skin protrusion usually located anterior to the anus [1] and $5.5-28.5 \mathrm{~mm}^{2}$ in size [3] that is covered by pink or light red skin and has a smooth surface [1]. However, some IPPPs described had either the shape of a peanut, were tongue-like [3] or leaf-like [5], and were located posterior to the anus [5] or presented concomitantly anterior and posterior to the anus [5, 7]. Due to this variety in the location and shape of IPPPs, some authors prefer the term infantile perineal protrusion $[3,9]$ and others the term infantile perianal protrusion [5]. 
The diagnosis of IPPP is based on physical examination [2]. Clinical findings of LSA in other parts of the body might mean that IPPP is a manifestation of LSA [10].

Histopathologic findings include acanthosis in the epidermis and dilated capillaries and elements of fibrous tissue infiltrated by eosinophils in the upper part of the dermis [1], but others have shown almost normal histological findings [3]. In the case of a LSArelated IPPP, histology shows patchy lichenoid infiltrates with vacuolar alteration and homogenization areas of the collage in the papillary dermis [9]. The differential diagnosis of IPPP includes sexual abuse, rectal prolapse, perianal lesions of Crohn's disease, hemangiomas, and hemorrhoid disease $[1,3,8,10]$.

A conservative approach is indicated in the constitutional type of IPPPs as in most cases, they might resolve only few weeks after initial presentation [1]. However, it has been noticed, as in patients 1-6 of this study, that this type of IPPP may remain unchanged over years [5]. Instructing parents to wipe the child's anal area from anterior to posterior may be helpful [1]. In the case of a coexisting constipation, appropriate treatment usually, but not always [5], results in the regression of the lesion $[6,7]$. IPPPs due to LSA can be treated with local corticosteroids [2, 9], although automatic regression has also been observed [9].

In conclusion, pediatric surgeons, pediatricians and other care givers should be aware of the lesion discussed. The characteristic morphology and location could help to avoid erroneous investigation of sexual abuse and rule out other types of lesions in this area. A detailed history regarding bowel habits may show associated constipation and an appropriate clinical examination may reveal signs of LSA. Moreover, awareness of IPPP may help to further elucidate the incidence, pathogenesis, and natural history of this lesion.

\section{Disclosure Statement}

The authors declare that there are no conflicts of interest. 
Table 1. Summary of the published articles on IPPPs in children

\begin{tabular}{lrccll}
\hline Articles & Patients & Age at diagnosis & $\begin{array}{l}\text { Presentation } \\
\text { at birth }\end{array}$ & $\begin{array}{l}\text { Male/ } \\
\text { female }\end{array}$ & Biopsy \\
\hline McCann et al., 1989 [4] & 18 & 2-10 years & - & $-/ 18$ & no \\
Kayashima et al., 1996 [1] & 15 & 11 months to 2 years & - & $1 / 14$ & $2 / 15:$ IPPP \\
Cruces et al., 1998 [9] & 4 & 7 months to 2 years & - & $-/ 4$ & $4 / 4:$ LSA \\
Mérigou et al., 1998 [7] & 4 & 6-20 months & - & $-/ 4$ & no \\
Konta et al., 2000 [3] & 36 & 1 day to 6 months & $14 / 36$ & $-/ 36$ & $4 / 36$ : IPPP \\
Patrizi et al., 2002 [5] & 13 & 7 months to 7 years & $2 / 13$ & $-/ 13$ & $1 / 13$ : LSA \\
Miyamoto et al., 2004 [11] & 1 & 11 months & - & $-/ 1$ & no \\
Fleet and Davis, 2005 [8] & 1 & 12 months & - & $-/ 1$ & no \\
Khachemoune, 2006 [12] & 2 & $4-9$ months & - & $-/ 2$ & no \\
Kim et al., 2007 [2] & 1 & 19 months & - & $-/ 1$ & LSA \\
Hernantez et al., 2007 [13] & 1 & 11 years & - & $-/ 1$ & LSA \\
Leung et al., 2010 [6] & 2 & 9 months to 1 year & - & $-/ 2$ & - \\
Haastrup et al., 2011 [14] & 1 & 13 years & - & $-/ 1$ & - \\
\hline
\end{tabular}

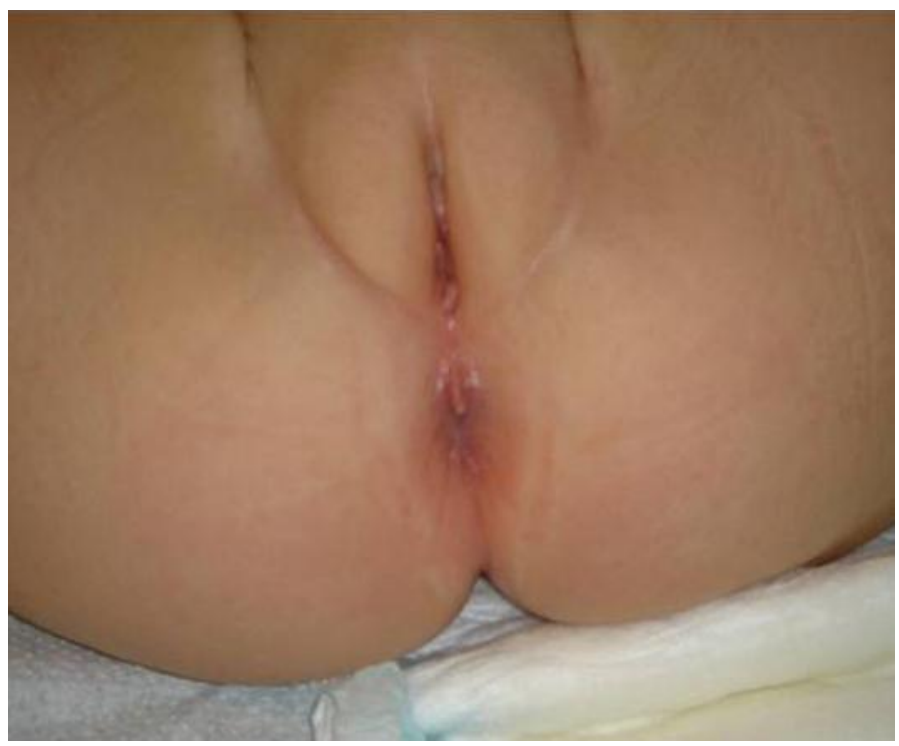

Fig. 1. A 6-month-old girl with a typical appearance of IPPP. 


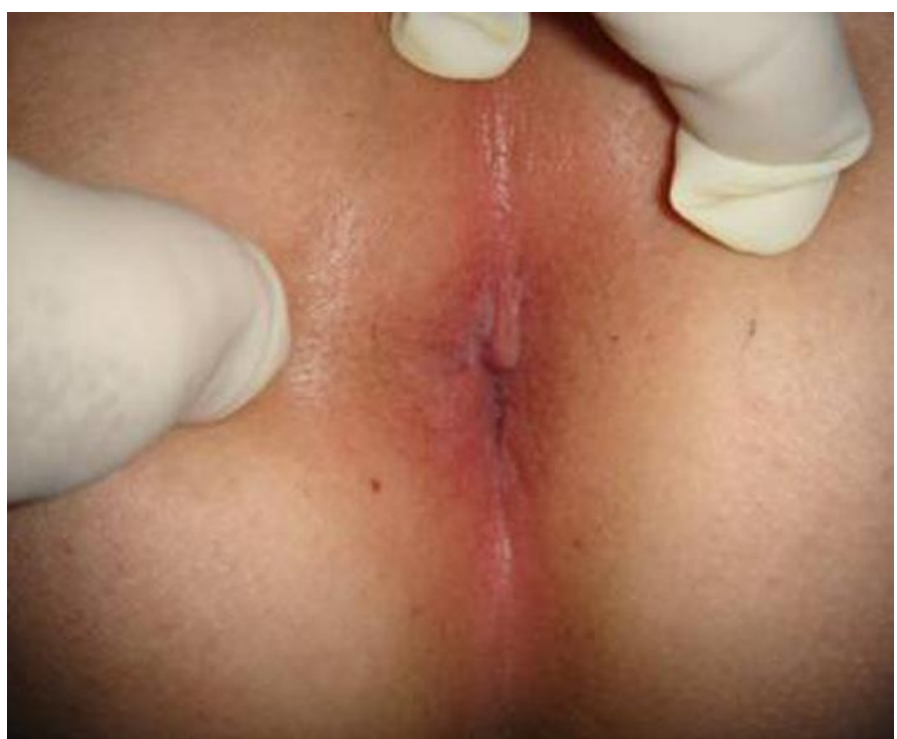

Fig. 2. A 12-year-old boy with a pyramidal protrusion anterior to the anus.

\section{References}

1 Kayashima K, Kitoh M, Ono T: Infantile perianal pyramidal protrusion. Arch Dermatol 1996;132:14811484.

2 Kim BJ, Woo SM, Li K, Lee DH, Cho S: Infantile perianal pyramidal protrusion treated by topical steroid application. J Eur Acad Dermatol Venereol 2007;21:263-264.

3 Konta R, Hashimoto I, Takahashi M, Tamai K: Infantile perineal protrusion. Dermatology 2000;201:316320.

4 McCann J, Voris J, Simon M, Wells R: Perianal findings in prepubertal children selected for non-abuse: a descriptive study. Child Abuse Negl 1989;13:179-193.

5 Patrizi A, Raone B, Neri I, D’Antuono A: Infantile perianal protrusion: 13 new cases. Pediatr Dermatol 2002;19:15-18.

-6 Leung AK: Concomitant anterior and posterior infantile perianal protrusions. J Natl Med Assoc 2010;102:135-136.

7 Mérigou D, Labrèze C, Lamireau T, Vergnes P, Taïeb A: Infantile perianal pyramidal protrusion: a marker of constipation? Pediatr Dermatol 1998;15:143-144.

$>8$ Fleet SL, Davis LS: Infantile perianal pyramidal protrusion: report of a case and review of the literature. Pediatr Dermatol 2005;22:151-152.

-9 Cruces ML, De La Torre C, Losada A, Ocampo C, García-Doval I: Infantile pyramidal protrusion as a manifestation of lichen sclerosus et atrophicus. Arch Dermatol 1998;134:1118-1120.

10 Tsunemi Y, Matsushita T, Takahashi T, Tamaki T: Childhood capillary hemangioma presenting as infantile perianal protrusion. Dermatology 2003;207:408-409.

11 Miyamato T, Inoue S, Hagari Y, Mihara M: Infantile perianal pyramidal protrusion with hard stool history. Br J Dermatol 2004;151:229.

12 Khachemoune A, Guldbakke KK, Ehrsam E: Infantile perineal protrusion. J Am Acad Dermatol 2006;54:1046-1049.

13 Hernandez-Machin B, Almeida P, Lujan D, Montenegro T, Borrego L: Infantile pyramidal protrusion localized at the vulva as a manifestation of lichen sclerosus et atrophicus. J Am Acad Dermatol 2007;56:S49-S50.

14 Haastrup MD, Bygum A: Infantile perineal protrusion is a harmless condition (in Danish). Ugeskr Laeger 2001;173:2571-2572. 